\title{
Análise de estresse operacional e fatores associados em trabalhadores de call center de Montes Claros-MG
}

\author{
Operational stress analysis and factors associated in workers of call center in Montes \\ Claros-MG
}
Análisis del estrés operativo y factores asociados en trabajadores del centro de llamadas de Montes Claros-MG

Fábio Ribeiro $^{1 *}$, Nathaly Silva Silveira ${ }^{1}$, Victória Silva Lidório ${ }^{1}$, Caroline Sousa Dias ${ }^{1}$, Joaquim Pereira de Melo Neto ${ }^{1}$, Pedro Rocha Gonçalves ${ }^{1}$, Bárbara Silva Lidório ${ }^{1}$, Daniel Oliva Brito ${ }^{1}$, Bianca Galvão Rodrigues ${ }^{1}$, Paulo Henrique Batista Amorim¹, Cristiano Alves de Souza1 .

\section{RESUMO}

Objetivo: Esse estudo teve como objetivo analisar o nível de estresse e fatores associados em operadores de Call Center de Montes Claros-MG. Métodos: O instrumento utilizado foi a Escala de Percepção do Estresse (PSS-14), a dosagem de glicemia capilar, aferição de pressão arterial e medidas da frequência cardíaca, peso, altura e circunferência abdominal. Também foram verificadas as relações com fatores socio laborais como sexo, idade, estado civil, escolaridade, experiência, turno, carga horária de trabalho, tabagismo e prática de atividade física. Participaram da pesquisa 363 operadores de empresas de Call Centers localizadas na cidade de Montes Claros. Resultados: Evidenciou-se que a maioria dos trabalhadores eram do sexo feminino, e que as mulheres foram mais suscetíveis ao estresse do que os homens. Também podese inferir que aqueles que praticavam atividade física possuíam menores níveis de estresse. Conclusão: De forma geral, os trabalhadores se apresentaram bastante tolerantes ao estresse laboral, o que nos leva a acreditar numa melhora da qualidade de trabalho que vem sendo implementada por diversos setores psicossociais em Call Centers. O cuidado com a saúde também se mostrou relevante, sendo importante reforçar que a prática de atividade física tem grande impacto na saúde física e mental.

Palavras-chave: Trabalhadores, Teste de Estresse, Estresse psicológico, Call Center.

\begin{abstract}
Objective: This study had as objective to analyze the level of stress and associated factors in Call Center of Montes Claros-MG. Methods: The instrument used for a Stress Perceptual Scale (PSS-14), a blood glucose measurement, blood pressure measurement and measures of heart rate, weight, height and waist circumference. They were also verified as relations with socio-labor factors such as sex, age, marital status, education, experience, shift, workload, smoking and physical activity. A total of 363 operators of Call Center companies located in the city of Montes Claros participated in the survey. Results: The results showed that most workers are female, and as women are more susceptible to stress than men. It can also be inferred that those who practiced physical activity had lower levels of stress. Conclusion: In general, the workers are presenters quite tolerant to work stress, which leads us to believe in the improvement of the quality of work that has been implemented by several psychosocial sectors in Call Centers. In addition, health care is also shown to be relevant, and it is important to reinforce that the practice of physical activity has a great impact on physical and mental health.
\end{abstract}

Keywords: Workers, Stress, Call Center, Mental Suffering.

${ }^{1}$ Centro Universitário FIPMoc (UNIFIPMoc), Montes Claros-MG. *E-mail: fabio.ribe@yahoo.com.br

SUBMETIDO EM: 10/2019

ACEITO EM: 10/2019

PUBLICADO EM: 12/2019 


\section{RESUMEN}

Objetivo: Este estudio tuvo como objetivo analizar el nivel de estrés y los factores asociados en los operadores de Call Center de Montes Claros-MG. Métodos: El instrumento utilizado fue la Escala de Percepción de Estrés (PSS-14), medición de glucosa en sangre capilar, medición de presión arterial y mediciones de frecuencia cardíaca, peso, altura y circunferencia de cintura. También se verificaron las relaciones con factores socio-ocupacionales como género, edad, estado civil, educación, experiencia, turno, carga de trabajo, tabaquismo y actividad física. En la encuesta participaron 363 operadores de empresas de call center ubicadas en la ciudad de Montes Claros. Resultados: se descubrió que la mayoría de los trabajadores eran mujeres y que las mujeres eran más susceptibles al estrés que los hombres. También se puede inferir que aquellos que practicaban actividad física tenían niveles de estrés más bajos. Conclusión: En general, los trabajadores eran muy tolerantes al estrés laboral, lo que nos lleva a creer en una mejora en la calidad del trabajo que han implementado varios sectores psicosociales en los Call Centers. La atención médica también fue relevante y es importante reforzar que la práctica de actividad física tiene un gran impacto en la salud física y mental.

Palabras clave: Trabajadores, Teste de Estrés, Estrés psicológico, Centro de llamadas,

\section{INTRODUÇÃO}

Os Call Centers, desde o seu surgimento no início dos anos 90, se tornaram peça chave no mundo dos negócios, servindo como primeiro canal de baixo custo, entre o cliente e a empresa. Podem ser definidos como um local de trabalho onde os agentes de serviço interagem com clientes, principalmente por meio do telefone, tendo como suporte os sistemas de computação (MOLINO M, et al.,2016).

Os Call Centers representam um setor econômico que, nos últimos anos, incorporou milhares de trabalhadores. As tarefas realizadas possuem características, potencialmente, comprometedoras da qualidade de vida de quem as realiza, pois lança mão de uso intensivo de tecnologia e se baseia em diferentes formas de controle sobre o desempenho do trabalhador, como a pressão por produtividade visando aumentar os atendimentos. Desconforto, rapidez, afobação, estresse, ansiedade e pressão para manter a qualidade são condições com que esses profissionais lidam, gerando prejuízo na qualidade de vida profissional (PARISE JA e SOLER ZASG, 2016).

Os trabalhadores de Call Center são em sua maioria jovens, mulheres, pessoas com mais de 40 anos ou pessoas com dificuldades de acesso ao mercado de trabalho. Na maioria dos casos é o primeiro emprego de muitas pessoas, que é caracterizado por baixa remuneração e poucas perspectivas de crescimento, o que justifica não ser exigida uma alta escolaridade. Há uma predominância de mulheres, cerca de $80 \%$, e uma rotatividade elevada, originando contratação constante. Dessa forma, sugere-se uma reflexão acerca das condições de trabalho e das relações existentes nessa organização laboral (ZILIOTTO DM e OLIVEIRA BO, 2014).

O stress relacionado ao trabalho pode ser definido como um conjunto de reações prejudiciais, tanto físicas como emocionais, que ocorrem quando existe um desequilíbrio entre as exigências do local de trabalho e a capacidade ou recursos do trabalhador. A literatura tem se apresentado bem enfática ao mostrar os efeitos adversos que o stress provoca na saúde humana. As doenças mais comumente associadas são: doenças cardiovasculares, desordens no sistema neuroendócrino que podem levar ao aumento substancial dos níveis de glicose no sangue, distúrbios da esfera psíquica, do sistema imunológico, gastrointestinal e até mesmo pele e músculos (SANCINI A, et al., 2017). A Escala de Percepção do Stress (PSS-14) é uma escala de avaliação objetiva, onde é medido o grau de estresse nas situações da vida de uma pessoa. É composta por 14 itens sob a forma de perguntas, respondidas em escala de 5 pontos (0-Nunca a 4-Sempre), com escore final entre 0 a 56 pontos (FARO A, 2015).

As doenças ateroscleróticas coronarianas são encontradas, frequentemente, em associação com o estresse no trabalho. Os principais fatores de risco para essas doenças são: pressão alta, perfil lipídico 
anormal, tabagismo e inatividade física, todos fatores evitáveis. Muitos adultos não possuem conhecimento sobre as doenças ateroscleróticas coronarianas e, por isso, não adotam um estilo de vida saudável para reduzir seus riscos. A doença causa perda econômica e de produtividade sob a população economicamente ativa, devendo, portanto, sempre ser evitada (ZHANG H, et al., 2017).

Dessa maneira, conhecimento sobre o índice do estresse em trabalhadores de Call Center auxiliará tanto empresas quanto trabalhadores, melhorando seu desempenho profissional e possibilitando a melhora na qualidade de vida. Esse estudo teve como objetivo analisar o nível de estresse e fatores associados em operadores de Call Center de Montes Claros-MG.

\section{MÉTODO}

Trata-se de um estudo de investigação, prospectivo, transversal, de caráter descritivo e quantitativo. Foi realizado em empresas de Call Center, na cidade de Montes Claros-MG, que possui atualmente 398.288 mil habitantes, no ano de 2017. Participaram desta pesquisa 363 operadores de Call Center de duas empresas, que contam com duas sedes cada, totalizando assim quatro locais diferentes de Call Centers, com uma média estimada de 6 mil operadores no total. De acordo com o sigilo proposto pelo nosso trabalho e pelo Comitê de Ética, as empresas e suas respectivas sedes foram denominadas como: Empresa $1=E 1$ e sua sede $E 1 \mathrm{~A}$, e Empresa 2 = E2 e sua sede E2B. A empresa denominada E1 e E1A trata-se de uma empresa de Call Center passivo, e a empresa E2 e E2B é do tipo ativo. Só foi validada a participação daqueles que concordaram voluntariamente em se submeter a todos os itens propostos pela pesquisa, que assinaram o Termo de Consentimento Livre e Esclarecido, e que no momento de realização da estavam em seu ambiente de trabalho. Foram excluídos os trabalhadores que não assinaram o Termo de Consentimento Livre e Esclarecido e os que estavam afastados de suas atividades por licença médica, licença maternidade ou férias.

Para a coleta de dados foi aplicado o questionário da Escala de Estresse Percebido (PSS-14) e questionário de dados sociodemográficos e laborais contendo cinco questões, elaborado pelos autores do estudo, com o objetivo de coletar dados sobre sexo, idade, escolaridade, estado civil, tempo de experiência na função, turno de trabalho, prática de atividade física e tabagismo. Também foram avaliados dados antropométricos (peso, altura, índice de massa corporal (IMC), circunferência abdominal CA)) e dados vitais (pressão arterial (PA), frequência cardíaca ( $F C$ ) e glicemia capilar).

A Escala de Percepção do Estresse - Perceived Stress Scale (PSS-14), é uma ferramenta de avaliação objetiva, que mede o grau em que situações na vida de alguém são tidas como estressantes. A escala é

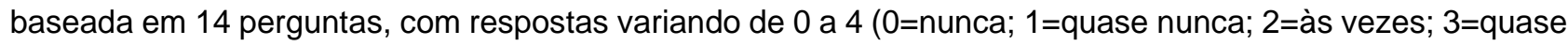
sempre; 4=sempre) para cada item, considerando sua ocorrência pelo menos um mês antes da aplicação da pesquisa. As questões com conotação positiva $(4,5,6,7,9,10$ e 13) têm sua pontuação somada invertida, da seguinte maneira, $0=4,1=3,2=2,3=1$ e $4=0$. As demais questões são negativas e devem ser somadas diretamente (LUFT CD et al., 2007).

O PSS-14 tem uma consistência interna de 0,85 (coeficiente $\alpha$ de Cronbach) e confiabilidade teste-reteste durante um curto intervalo de reteste (vários dias) de 0,85. O PSS-14 tem uma gama possível de pontuações de 0 a 56, sendo 28 o valor considerado como operacional de corte (CHOWDHURY R, et al., 2017).

Para a avaliação desses dados foram usados: Esfigmomanômetro da marca BD, Estetoscópio da marca Littmann ${ }^{\circledR}$, Balança Digital Techline ${ }^{\circledR}$, Fita Métrica Flexível de 1,5 metros, Glicosímetro da marca Accu-chek ${ }^{\circledR}$ e Fitas de glicosímetro. A coleta de dados foi realizada no próprio local de trabalho dos participantes, abrangendo os turnos: matutino, vespertino e noturno, durantes suas pausas no serviço ou na saída.

Para caracterizar o universo amostral pesquisado foi utilizada uma análise descritiva através de porcentagem, média e desvio padrão, mediante fórmula de cálculo amostral, que nos forneceu uma amostra necessária de 363 entrevistados, com 5\% de erro amostral e 95\% de nível de confiança, para uma população de 6000 pessoas. Para identificação do perfil socioeconômico, demográfico e antropométrico foram utilizados frequência, porcentagem, média e desvio padrão, e para comparação entre as variáveis do estudo foi empregado a ferramenta estatística Software Statistical Package for Social Sciences 18.0 (SPSS) por meio 
de testes paramétricos e não-paramétricos. Para as análises descritivas, os resultados estão apresentados em frequências absolutas e relativas e em medidas de tendência central e variabilidade, como média e desviopadrão. Nas análises bivariadas das variáveis categóricas foi utilizado o Teste de Qui-Quadrado de Pearson e, como alternativa, o Teste Likelihood Ratio nos casos em que o Qui-Quadrado não era recomendado. Nas análises bivariadas das variáveis numéricas foi utilizado o Teste t de Student, pois, as mesmas apresentavam distribuição normal. Esse estudo foi aprovado pelo Comitê de Ética das Faculdades Integradas Pitágoras (FIP-MOC) processo número 2.100.390.

\section{RESULTADOS}

A análise descritiva de características relativas aos trabalhadores de Call Centers demonstrou que os resultados obtidos de 363 participantes $65,3 \%(\mathrm{~N}=237$ ) eram do sexo feminino enquanto $34,7 \%(\mathrm{~N}=126)$ eram do sexo masculino. A maioria dos operadores $77,7 \%(\mathrm{~N}=282)$ era composta por solteiros (Tabela 1).

Tabela 1 - Análise descritiva de características relativas aos trabalhadores de Call Centers em Montes Claros (MG).

\begin{tabular}{|c|c|c|}
\hline Variáveis & $\mathbf{n}$ & $\%$ \\
\hline \multicolumn{3}{|l|}{ PSS - Estresse } \\
\hline Menos estressado (<28 PSS) & 189 & 52,1 \\
\hline Mais estressado ( $>=28$ PSS) & 174 & 47,9 \\
\hline \multicolumn{3}{|l|}{ Sexo } \\
\hline Feminino & 237 & 65,3 \\
\hline Masculino & 126 & 34,7 \\
\hline \multicolumn{3}{|l|}{ Idade } \\
\hline$<=20$ & 85 & 23,4 \\
\hline $21-30$ & 224 & 61,7 \\
\hline $31-40$ & 45 & 12,4 \\
\hline$>40$ & 9 & 2,5 \\
\hline \multicolumn{3}{|l|}{ Escolaridade } \\
\hline 5 a 8 anos & 194 & 53,4 \\
\hline$>$ de 8 & 169 & 46,6 \\
\hline \multicolumn{3}{|l|}{ Estado Civil } \\
\hline Solteiro & 282 & 77,7 \\
\hline Casado & 75 & 20,7 \\
\hline Outro & 6 & 1,7 \\
\hline \multicolumn{3}{|l|}{ Tempo trabalho } \\
\hline 1 a 6 meses & 98 & 27,0 \\
\hline 7 a 12 meses & 97 & 26,7 \\
\hline 13 meses a 3 anos & 110 & 30,3 \\
\hline$>$ de 3 anos & 58 & 16,0 \\
\hline \multicolumn{3}{|l|}{ Carga Horária } \\
\hline 6 horas & 324 & 89,3 \\
\hline 8 horas & 35 & 9,6 \\
\hline 10 horas & 4 & 1,1 \\
\hline \multicolumn{3}{|l|}{ Turno } \\
\hline Mat & 123 & 33,9 \\
\hline Vesp & 216 & 59,5 \\
\hline Not & 24 & 6,6 \\
\hline \multicolumn{3}{|l|}{ Atividade Física } \\
\hline Sim & 119 & 32,8 \\
\hline Não & 244 & 67,2 \\
\hline \multicolumn{3}{|l|}{ Tabagismo } \\
\hline Sim & 57 & 15,7 \\
\hline Não & 306 & 84,3 \\
\hline
\end{tabular}

Legenda: PSS- Estresse: Escala de Estresse Percebido; Mat- Matutino; Vesp- Vespertino; Not- Noturno;

Fonte: Ribeiro F, et al., 2019. 
A maior parte da população estudada $(61,7 \%)$ foi de jovens com idade variando entre $21-30$ anos, sendo a pessoa mais nova com 18 anos e a mais velha com 50 anos. O perfil de escolaridade mostrou que $52,6 \%$ dos participantes tinham apenas ensino médio completo contra $47,4 \%$ que estão frequentando ou possuem graduação. Em relação às características profissionais, 53,7\% trabalham há menos de 1 ano e apenas 16\% trabalham há mais de 3 anos. Outra consideração é que $89,3 \%$ dos indivíduos trabalham com a carga horária de serviço de 6 h e $20 \mathrm{~min}$ diárias, sendo a maioria $59,5 \%(\mathrm{~N}=216)$ trabalhando em turno vespertino, com intervalos de 10 minutos chamada "pausa lanche" para situações emergenciais e 20 minutos para "pausa almoço". Os que possuíam carga horária de $8 \mathrm{~h}$ e $20 \mathrm{~min}$ eram operadores que foram promovidos supervisores, perfazendo $9,6 \%$ da amostra $(\mathrm{N}=32)$ (Tabela 1).

A experiência na função avaliada, variou de 1 mês a 6 anos, o que não variou significativamente entre as categorias, pois apresentaram valores muitos próximos. A maioria encontrava-se na faixa entre 13 meses a 3 anos, 30,3\% ( $\mathrm{N}=110)$, contra 27\% ( $\mathrm{N}=98)$ de 1 mês a 6 meses, e $26,7 \%(\mathrm{~N}=97)$ de 7 a 12 meses. O turno de trabalho com o maior número de operadores foi o vespertino com $59,5 \%(\mathrm{~N}=216)$ (Tabela 1).

Quanto aos dados relacionados à saúde desses funcionários, obteve-se que a maioria não fazia prática de atividade física $67,2 \%(\mathrm{~N}=244)$. A maior parcela da amostra não era tabagista, representada por $84,3 \%$ $(\mathrm{N}=306)$ dos operadores (Tabela 1).

Em relação às variáveis físicas/fisiológicas, o estudo demonstrou que a pressão arterial diastólica aferida variou na faixa de 60-120 mmHg, enquanto a pressão arterial sistólica variou de 90-170 mmHg, sendo a média de $117 \times 76 \mathrm{mmHg}$. A medida de glicemia capilar variou de 46 a $337 \mathrm{mg} / \mathrm{dL}$, com uma média de $95 \mathrm{mg} / \mathrm{dL}$. $\mathrm{O}$ IMC variou de 16,8 a 42,45, sendo a média de 24,8. A frequência cardíaca variou entre 56 bpm a 126 bpm, com uma média de 84bpm (Tabela 2).

Tabela 2 - Descrição das variáveis de características físicas/fisiológicas dos trabalhadores de Call Centers em Montes Claros (MG).

\begin{tabular}{lcccc}
\hline Variáveis & Média & Desvio-Padrão & Mínimo & Máximo \\
\hline IMC & 24,80 & 4,60 & 16,80 & 42,45 \\
CA (cm) & 77,52 & 12,33 & 53,00 & 124,00 \\
FC (bpm) & 84,18 & 12,81 & 56,00 & 126,00 \\
PAS (mmHg) & 117,69 & 11,25 & 90,00 & 170,00 \\
PAD (mmHg) & 76,82 & 8,32 & 60,00 & 120,00 \\
GC (mg/dL) & 95,67 & 26,98 & 46,00 & 337,00 \\
\hline
\end{tabular}

Legenda: IMC: Índice de Massa Corporal; CA: Circunferência abdominal; FC: Frequência cardíaca; PAS: Pressão Arterial Sistólica; PAD: Pressão Arterial Diastólica; GC: Glicemia Capilar;

Fonte: Ribeiro F, et al., 2019.

A Tabela 3 apresenta a análise bivariada de fatores antropométricos associados ao estresse, demonstrando que entre os homens, a pressão arterial sistólica foi estatisticamente significativa maior nos mais estressados $(p=0,031)$. As mulheres menos estressadas apresentaram FC maior no grupo considerado menos estressado $(p<0,049)$.

Em relação à comparação entre os dados sócio laborais e a geração de estresse, o sexo feminino mostrouse mais estressado do que o masculino, com um valor de $55,3 \%$ entre as mulheres, contra $34,1 \%$ entre os homens $(p<0,001)$. A faixa etária composta por operadores menores de 20 anos foi a que apresentou maior número de estressados $(52,9 \%)$. Contudo, não houve diferença estatística quando comparada às diferentes faixas etárias avaliadas. 
Tabela 3 - Análise bivariada de fatores antropométricos por sexo associados ao estresse (PSS-14) em trabalhadores de Call Centers em Montes Claros (MG).

\begin{tabular}{lccc}
\hline Variáveis & Menos estressado N (DP*) & Mais Estressado N (DP*) & p-valor \\
\hline Mulher & & & \\
\hline IMC & $25,22(4,98)$ & $24,68(4,71)$ & 0,397 \\
CA (cm) & $76,73(13,9)$ & $75,56(11,2)$ & 0,477 \\
FC (bpm) & $87,85(12,32)$ & $84,65(12,4)$ & 0,049 \\
PAS (mmHg) & $116,11(10,38)$ & $114,01(10,27)$ & 0,120 \\
PAD (mmHg) & $75,28(7,83)$ & $74,96(7,06)$ & 0,740 \\
GC (mg/dL) & $96,01(31,89)$ & $98,88(27,37)$ & 0,457 \\
\hline Homem & & & \\
\hline IMC & $24,75(4,21)$ & $24,24(3,96)$ & 0,511 \\
CA (cm) & $80,8(11,63)$ & $79,14(11,76)$ & 0,452 \\
FC (bpm) & $79,47(12,08)$ & $82,77(13,9)$ & 0,170 \\
PAS (mmHg) & $121,33(10,33)$ & $125,81(12)$ & 0,031 \\
PAD (mmHg) & $79,04(7,43)$ & $81,98(11,29)$ & 0,128 \\
GC (mg/dL) & $92,23(18,62)$ & $91,72(25,73)$ & 0,899 \\
\hline
\end{tabular}

Legenda: IMC: Índice de Massa Corporal; CA: Circunferência abdominal; FC: Frequência cardíaca; PAS: Pressão Arterial Sistólica; PAD: Pressão Arterial Diastólica; GC: Glicemia Capilar; $\left(\mathrm{DP}^{\star}\right)$ : desvio padrão.

Fonte: Ribeiro F, et al., 2019.

A variável escolaridade também apresentou resultados interessantes, pois quanto menores os níveis de escolaridade maior o estresse comparativo na amostra, como demonstrado pelos $52,4 \%$ de 5 a 8 anos contra os $42,6 \%$ mais de 8 anos de escolaridade.

Tabela 4 - Análise bivariada dos fatores associados ao estresse (PSS-14) em trabalhadores de Call Centers em Montes Claros (MG).

\begin{tabular}{|c|c|c|c|}
\hline VARIÁVEIS & Menos estressado N (\%) & Mais Estressado N (\%) & $p$-valor \\
\hline \multicolumn{4}{|l|}{ Sexo } \\
\hline Feminino & $106(44,7)$ & $131(55,3)$ & \\
\hline Masculino & $83(65,9)$ & $43(34,1)$ & 0,001 \\
\hline \multicolumn{4}{|l|}{ Idade } \\
\hline$<=20$ & $40(47,1)$ & $45(52,9)$ & \\
\hline $21-30$ & $119(53,1)$ & $105(46,9)$ & \\
\hline $31-40$ & $24(53,3)$ & $21(46,7)$ & \\
\hline$>40$ & $6(66,7)$ & $3(33,3)$ & $0,622^{*}$ \\
\hline \multicolumn{4}{|l|}{ Escolaridade } \\
\hline 5 a 8 anos & $91(47,6)$ & $100(52,4)$ & \\
\hline Mais de 8 & $97(57,4)$ & $72(42,6)$ & $0,145^{\star}$ \\
\hline \multicolumn{4}{|l|}{ Estado Civil } \\
\hline Solteiro & $149(52,8)$ & $133(47,2)$ & \\
\hline Casado & $38(50,7)$ & $37(49,3)$ & \\
\hline Outro & $2(33,3)$ & $4(66,7)$ & $0,612^{*}$ \\
\hline \multicolumn{4}{|c|}{ Tempo de experiência } \\
\hline 1 a 6 meses & $50(51)$ & $48(49)$ & \\
\hline 7 a 12 meses & $45(46,4)$ & $52(53,6)$ & \\
\hline $13 \mathrm{~m}$ a 3 anos & $59(53,6)$ & $51(46,4)$ & \\
\hline Mais de 3 anos & $35(60,3)$ & $23(39,7)$ & 0,392 \\
\hline \multicolumn{4}{|l|}{ Carga Horária } \\
\hline $6 h$ & $168(51,2)$ & $160(48,78)$ & \\
\hline $8 h$ & $21(60)$ & $14(40)$ & 0,291 \\
\hline \multicolumn{4}{|l|}{ Atividade Física } \\
\hline Sim & $74(62,2)$ & $45(37,8)$ & \\
\hline Não & $115(47,1)$ & $129(52,9)$ & 0,007 \\
\hline \multicolumn{4}{|l|}{ Tabagismo } \\
\hline Sim & $28(49,1)$ & $29(50,9)$ & \\
\hline Não & $161(52,6)$ & $145(47,4)$ & 0,628 \\
\hline
\end{tabular}


Em relação ao local de trabalho como fator indutor de estresse, observou-se significativa diferença entre uma das empresas e sua respectiva sede. A sede da empresa denominada E2b, foi a que apresentou maiores índices de operadores estressados, com aproximadamente $64 \%$ da amostra, e com uma diferença estatisticamente significativa $(p<0,018)$ quando comparada com a outra sede E2. Para as demais sedes, não se observou diferenças relevantes.

Tabela 5 - Análise bivariada entre local de trabalho e ocorrência de estresse (PSS-14) em trabalhadores de Call Centers em Montes Claros (MG).

\begin{tabular}{lccr}
\hline VARIÁVEIS & Menos estressado N (\%) & Mais Estressado N (\%) & p-valor \\
\hline Q1 - Local & & & \\
\hline E1 & $100(54,1)$ & $85(45,9)$ & \\
E1a & $14(48,3)$ & $15(51,7)$ & \\
E2b & $53(60,9)$ & $34(39,1)$ & \\
\hline E2b & $22(35,5)$ & $40(64,5)$ & 0,018 \\
\hline
\end{tabular}

Legenda: E1: empresa 1; E1a: empresa 1a; E2: empresa 2; E2b: empresa E2b;

Fonte: Ribeiro F, et al., 2019.

\section{DISCUSSÃO}

Esse estudo visou relacionar o perfil antropométrico e sociodemográfico dos operadores de Call Center com o seu nível de estresse. Ziliotto DM e Oliveira BO (2014) apontam que os trabalhadores de Call Center são predominantemente jovens, em seu primeiro emprego, com pouca exigência de escolarização e dominado pelo público feminino, representando $80 \%$ dos operadores. Esses resultados são coerentes com os obtidos nesse estudo. Existem diversas jornadas de trabalho nos Call Centers, sendo a mais comum delas a de 180 horas mês, na qual o operador trabalha 6 dias por semana, folga 1 dia. Trabalha-se 6 horas e 20 minutos por dia, com uma pausa de 5 minutos para necessidades fisiológicas e 10 minutos para o lanche. Em busca de maximizar a produtividade em uma jornada de 6 horas, a empresa espera que o operador fale por 5 horas e 45 minutos (DUTRA N, et al., 2016).

O principal objetivo desse estudo foi avaliar a vulnerabilidade ao estresse em operadores de Call Center, utilizando como ferramenta a Escala de Stress Percebido PSS-14. De modo geral, a média de estresse entre os operadores de Call Centers foi de 26,9. Ao comparar esse índice com os resultados de Soares et al. (2017), sobre níveis de estresse em outras profissões, o nosso grupo avaliado fica abaixo apenas do grupo de mestrando e doutorandos (índice de PSS 29,10). O nível de estresse nos operadores de Call Centers é mais alto do que em profissões que envolvem vidas e grandes cargas de pressão como médicos, enfermeiros, policiais militares e cuidadores de idosos. Chowdhury $R$ et al. (2017) avaliaram o nível de estresse em estudantes de medicina e obtiveram como média o valor de PSS-14 de 29,58.

Cristofoletti MF et al. (2006) encontraram uma prevalência de sobrepeso em trabalhadores que referiram ganhar peso após trabalhar como operador de $31,6 \%$, e de obesos de $12,6 \%$. Entre os motivos relatados como relacionados ao aumento de peso foram: trabalho predominantemente sedentário, horários de lanches diferentes a cada dia, estresse do trabalho e beliscar alimentos. Nesse estudo, o IMC e a CA não tiveram variação relevante entre os homens e mulheres mais estressados e menos estressados, sendo a população considerada como normal. Dessa forma, os operadores não apresentaram aumento dos fatores de risco cardiovasculares, que seria o IMC acima de 25 considerado como valor definidor de sobrepeso, e a CA maior que $88 \mathrm{~cm}$ em mulheres e maior que $102 \mathrm{~cm}$ em homens.

O valor de glicemia casual para se considerar diabetes pela Diretriz da Sociedade Brasileira de Diabetes (2016-2017) é acima de $200 \mathrm{mg} / \mathrm{dl}$ com sintomas clássicos. Nos operadores avaliados nesse estudo, a glicemia capilar estava dentro dos valores da normalidade. Podemos relacionar esse valor com o fato de os operadores terem poucos momentos para se alimentar, saindo do trabalho, quando eram abordados por nós, após um longo período de jejum. A média de pressão arterial sistólica e diastólica da amostra estudada estava dentro dos valores normais definidos pela Sociedade Brasileira de Cardiologia (2016), definida como 120x80 $\mathrm{mmHg}$. Apenas uma pequena amostra de homens mais estressados, se encontrava com PAS de 125,81 
$\mathrm{mmHg}$. Contudo, a maioria dos operadores avaliados estava com a frequência cardíaca dentro dos padrões de normalidade.

Rocha JC e Araújo GF (2016) descrevem que o nível de estresse em pessoas do sexo feminino vem aumentando nos últimos anos tornando-se maior quando comparado ao sexo masculino. Esse dado é corroborado por esse estudo, uma vez que encontramos o nível de estresse maior em mulheres. Esse fato pode ser explicado pela sobrecarga de atividade, pois as mulheres têm de lidar com sua carreira (profissional e acadêmica) e outras exigências cotidianas seja de cunho pessoal, sexual ou biológico. Esse fato pode explicar a inatividade física mais elevada entre as mulheres. Segundo Farah BQ et al. (2013), o maior envolvimento das mulheres em afazeres domésticos em adição à jornada de trabalho acaba reduzindo a disponibilidade de tempo para envolvimento em atividades físicas e de lazer.

Em relação à idade, percebe-se que não há relação significativa do nível de estresse e a idade, o que se observa é uma leve diminuição do estresse com o aumento da idade, sendo um pouco mais alto nos indivíduos mais jovens. Essa evidência é demonstrada na literatura em Lima FB (2004), Suehiro AC (2008), Dantas MA (2010), Costa DT (2011), Rodrigues VM (2011) que apesar de também não terem encontrado relação estatisticamente significativa, demonstraram níveis de estresse maior em trabalhadores mais jovens em diversos ramos de emprego como enfermeiros, policiais e mesmo no próprio operador de telemarketing. As mesmas referências sugerem que a capacidade de enfrentamento de uma situação estressora pode estar relacionada com a maturidade do indivíduo, e os sujeitos mais velhos seriam mais experientes e possuem um repertório mais variado para enfrentar essas situações.

A idade mais jovem, o sexo feminino, estado civil solteiro, escolaridade mais baixa e menor tempo na empresa parecem estar mais relacionados com o estresse segundo a literatura. Apesar de não haver significância estatística, muitos estudos apresentam dados semelhantes aos encontrados nesse estudo (BUBLITZ S 2016; LIMA FB 2004; PERTALI GB 2015; COSTA DT e MARTINS MCF, 2011). A associação da variável menor tempo na empresa e estresse poderia ser explicada pela falta de experiência no cargo que gera ansiedade e "medo de errar". O estado civil parece influenciar o estresse. Moraes LFR (2003) argumentam que o fato de os trabalhadores serem casados e possuírem uma estrutura familiar estabelecida, proporciona maiores momentos de prazer e maior equilíbrio pessoal por serem relações de companheirismo e cumplicidade.

O presente estudo não detectou diferença significativa entre operadores mais ou menos estressados e o hábito de fumar. Esse achado está em desacordo com o encontrado por Koltermann ITA (2011), Sousa V (2015) e Azevedo BDS (2017) que demonstram que o estresse muitas vezes está associado ao tabagismo. Esse resultado poderia ser explicado pelo número reduzido (15,7\%) de fumantes entre os operadores dos Call Centers avaliados. Com ações de controle do tabaco, a prevalência de tabagismo vem diminuindo ao longo dos anos no Brasil. O percentual de fumantes de 18 anos ou mais no país era de $34,8 \%$ em 1989. Esse número caiu para $14,7 \%$ em 2013 , de acordo com pesquisa para essa mesma faixa etária para áreas urbanas e rurais. (PNS, 2014).

Uma associação com relevância estatística foi a relação de estresse e ausência de atividade física. Esse estudo demonstrou que pessoas que praticam atividade física são menos estressadas $(p<0,007)$. A atividade física além de diminuir o risco de doenças cardiovasculares, é uma opção útil associada ao controle de estresse Soares MB, et al., (2017); Farah BQ, et al., (2013). Dos Santos Cavalcanti JOF, et al. (2016), propõem a introdução da ginástica laboral, uma atividade não nova, porém pouco aplicada, mas que vem ganhando espaço em países de primeiro mundo como Polônia, Japão e Holanda. As primeiras manifestações de atividades físicas entre funcionários são de 1901, mas somente em 1973 a ginástica laboral teve sua proposta inicial publicada no Brasil. Como já citado, o operador de telemarketing está em nível de estresse maior quando comparado com a maioria das profissões. A prática de atividade física nesse grupo se mostra essencial.

O operador pode ser do tipo ativo, quando a empresa que busca o contato com o cliente, ou receptivo, quando o cliente é que busca o contato com a empresa, e para isso o operador precisa de maior conhecimento 
do produto/serviço, ou de maior esforço para atingir as metas estabelecidas (TAKAHASHI LT, et al., 2014). Nesse sentido percebeu-se que a empresa do tipo receptiva apresentou uma porcentagem maior de operadores estressados, o que pode ser explicado pela dificuldade de resolução de problemas dos clientes que já ligam agressivos e impacientes. A diferença significativa do estresse entre os operadores de uma mesma empresa detectado nesse estudo pode ser devido às diferenças de infraestrutura fornecida, por exemplo: ar condicionado, espaço por metro quadrado, janelas, acessibilidade, banheiros e localização da sede.

Por esse estudo se tratar de um delineamento transversal, não foi possível acompanhar os profissionais e realizar análises relativas ao aumento ou diminuição do estresse com o passar do tempo de serviço. Apesar de poucos estudos correlacionando fatores antropométricos, sociodemográficos e estresse em operadores de Call Centers, esse estudo demonstrou que este grupo se encontra enquadrado dentro de trabalhadores mais estressados, e que a prática de atividade física se mostrou relevante para o grupo.

\section{CONCLUSÃO}

Assim, faz-se necessário investir em políticas de valorização do operador com ações que visem cuidar da saúde mental e física destes, pois se trata de um tipo de trabalho desgastante e estressante, tanto por trabalharem sob pressão constante para atingir metas como por solucionar problemas de clientes. 0 abandono a vida sedentária deve ser mais bem difundido para toda a população, principalmente se tratando de grupos economicamente ativos, que irão refletir diretamente na longevidade e em um envelhecimento mais saudável, com menor morbidade por fatores evitáveis. A melhoria nas condições de serviço será útil, tanto para o empregado quanto para o empregador, visto que um trabalhador menos estressado pode render mais, diminui o absenteísmo por afecções e gera melhor qualidade de vida para este profissional.

\section{REFERÊNCIAS}

1. AZEVEDO BDS, et al. Estresse Ocupacional E Insatisfação Com A Qualidade De Vida No Trabalho Da Enfermagem. Texto Contexto em Enfermagem. v. 26, n. 1. 2017.

2. BRASIL. Instituto Brasileiro de Geografia e Estatística. Pesquisa Nacional de Saúde. Rio de Janeiro: IBGE, 2014.

3. BUBLITZ S, et al. Associação Entre Estresse E Características Sociodemográficas E Acadêmicas De Estudantes De Enfermagem. Texto Contexto em Enfermagem. v. 25, n. 4. 2016.

4. CHOWDHURY R, et al. Perceived psychological stress among undergraduate medical students: Role of academic factors. Indian of Journal Public Health, v. 61, n, p. 155-57, Jan-Mar. 2017.

5. COSTA DT, MARTINS MCF. Estresse em profissionais de enfermagem: impacto do conflito no grupo e do poder do médico. Revista da Escola de Enfermagem da USP. v. 45, n. 5, p. 11918. 2011.

6. CRISTOFOLETTI MF, et al. Prevalência de sobrepeso, obesidade e obesidade abdominal em operadores de duas centrais de atendimento telefônico de São Paulo. Saúde, Ética \& Justiça. São Paulo, v. 11, n. 1-2, p. 19-28, nov. 2006.

7. DANTAS MA. Avaliação de estresse em policiais militares. Psicologia teoria e prática. v.12, n. 3. São Paulo mar. 2010.

8. DOS SANTOS CAVALCANTI JOF, et al. A prática da ginástica laboral e da atividade física como meio de melhoria dos sintomas das doenças ocupacionais. Revista Campo do Saber. v. 1, n. 1, 2016.

9. DUTRA N, et al. Estudo crítico sobre o trabalho em center. Revista Gestão \& Conexões. v. 5, n. 1, p. 38-66, 2016.

10. FARAH BQ, et al. Percepção de estresse: associação com a prática de atividades físicas no lazer e comportamentos sedentários em trabalhadores da indústria. Revista Brasileira de Educação Física e Esporte, São Paulo, v. 27, n. 2, p. 225-34, Abr-jun. 2013.

11. FARO A. Análise fatorial confirmatória das três versões da Perceived Stress Scale (PSS): um estudo populacional. Psicologia Reflexão e crítica. Porto Alegre, v. 28, n. 1, p.21-30, Mar. 2015.

12. KOLTERMANN ITA, et al. Estresse ocupacional em trabalhadores bancários: prevalência e fatores associados. Saúde (Santa Maria), A head of Print. v.37, n.2, p. 3348, 2011.

13. LIMA FB. Stress, Qualidade de Vida, Prazer e Sofrimento no Trabalhador de Call Center. 133f. Dissertação (Mestrado em Psicologia) -Centro de Ciências da Vida. PUC-Campinas. Campinas. 2004.

14. LUFT CDB, et al. Versão brasileira da Escala de Estresse Percebido: tradução e validação para idosos. Revista de Saúde Pública, São Paulo, v. 41, n. 4, p. 606-615. 2007. 
15. MOLINO M, et al. Inbound Call Centers and Emotional Dissonance in the Job Demands - Resources Model. Frontiers in Psychology, v. 7 p.1133. 2016.

16. MORAES LFR, et al. Qualidade De Vida No Trabalho E Estresse Ocupacional Na Polícia Militar De Minas Gerais. Revista de Psicologia: Saúde Mental e Segurança Pública. Belo Horizonte, v. 3, p. 53-58, jan./dez. 2003.

17. PARISE JA, SOLER ZASG. Quality of working life of call-center workers. Revista Brasileira de Enfermagem, v. 69 , n. 4, p. 704-709. 2016.

18. PERTALI GB, et al. Estresse ocupacional e fatores associados em trabalhadores bancários, Vitória - ES, Brasil. Ciência \& Saúde Coletiva, v. 20, n. 12, p.3925-3934. 2015.

19. ROCHA JC, ARAÚJO GF. Percepção do estresse em operadoras de telemarketing. Id on Line Revista Multidisciplinar e de Psicologia. v.10, n. 32. nov-dez. 2016.

20. RODRIGUES VMCP, et al. Fatores geradores de estresse em enfermeiros de Unidades de Terapia Intensiva. Revista Latino-Americana de Enfermagem. v.19, n.4. Ribeirão Preto Jul-ago. 2011.

21. SANCINI A, et al. Work-related stress and blood glucose levels. Ann Ig, v. 29, n. 2, p.123-133, mar-abr. 2017.

22. SOARES MB, et al. Escala de Estresse Percebido aplicada a docentes da Universidade Federal De Viçosa: Um estudo de validação de instrumento. Enangrad. Brasília, ago. 2017.

23. SOARES JP, et al. Qualidade de Vida, Estresse, Nível de Atividade Física e Cronotipo dos Auxiliares/Técnicos de Enfermagem em Unidades de Pronto Atendimento em Palmas/TO. Revista CPAQV - Centro de Pesquisas Avançadas em Qualidade de Vida. v. 9, n. 1. 2017.

24. Sociedade Brasileira de Cardiologia. 7ํㅡㄹ Diretriz Brasileira de Hipertensão Arterial. v. 107, № 3, Supl. 3, set. 2016.

25. Sociedade Brasileira de Diabetes. Diretrizes da Sociedade Brasileira de Diabetes. 2015-2016.

26. SOUSA V, et al. Estresse Ocupacional E Qualidade De Vida De Profissionais Da Limpeza Urbana. Revista de Saúde Pública de Santa Catarina. Florianópolis, v. 8, n. 2, p. 8-20, maio/ago. 2015;

27. SUEHIRO ACB, et al. Vulnerabilidade ao estresse e satisfação no trabalho em profissionais do Programa de Saúde da Família. Boletim de psicologia. v.58, n.129 São Paulo dez. 2008.

28. TAKAHASHI LT, et al. Avaliação da vulnerabilidade ao estresse no trabalho de operadores de Telemarketing. Revista de Psicologia, Organização e Trabalho. Florianópolis, v. 14, n. 3, p. 336-346, set. 2014.

29. ZHANG $\mathrm{H}$, et al. The effect of a smartphone-based coronary heart disease prevention (SBCHDP) programme on awareness and knowledge of CHD, stress, and cardiac-related lifestyle behaviours among the working population in Singapore: a pilot randomised controlled trial. Health and Quality of Life Outcomes, v.14;15, n. 1, p. 49, mar. 2017.

30. ZILIOTTO DM, OLIVEIRA BO. A organização do trabalho em call centers: implicações na saúde mental dos operadores. Revista Psicologia: Organizações e Trabalho, v. 14, n. 2, p. 169-179, 2014. 